\title{
Global responses to tris(1-chloro-2-propyl)phosphate (TCPP) in rockfish Sebastes schlegeli using integrated proteomic and metabolomic approach
}

\section{Chenglong Ji ${ }^{\text {a,b,c }}$, Zhen $\mathrm{Lu}^{\mathrm{a}, \mathrm{d}}$, Lanlan $\mathrm{Xu}^{\mathrm{a}, \mathrm{d}}$, Fei $\mathrm{Li}^{\mathrm{a}, \mathrm{c}}$, Ming Cong ${ }^{\mathrm{a}, \mathrm{c}}$, Xiujuan Shan ${ }^{\mathrm{b}}$, Huifeng Wu ${ }^{\mathrm{a}, \mathrm{b}, \mathrm{c}, *}$}

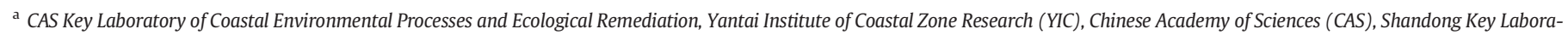
tory of Coastal Environmental Processes, YICCAS, Yantai 264003, PR China

b Laboratory for Marine Fisheries Science and Food Production Processes, Qingdao National Laboratory for Marine Science and Technology, Qingdao 266237, PR China

c Center for Ocean Mega-Science, Chinese Academy of Sciences (CAS), Qingdao 266071, PR China

' University of Chinese Academy of Sciences, Beijing 100049, PR China

\section{H I G H L I G H T S}

- The proteome and metabolome responses were identified in rockfish treated with TCPP.

- A hypothesized network of proteins and metabolites in rockfish was summarized.

- The alterations of nervous system related proteins suggested neurotoxicity of TCPP.

\section{G R A P H I C A L A B S T R A C T}

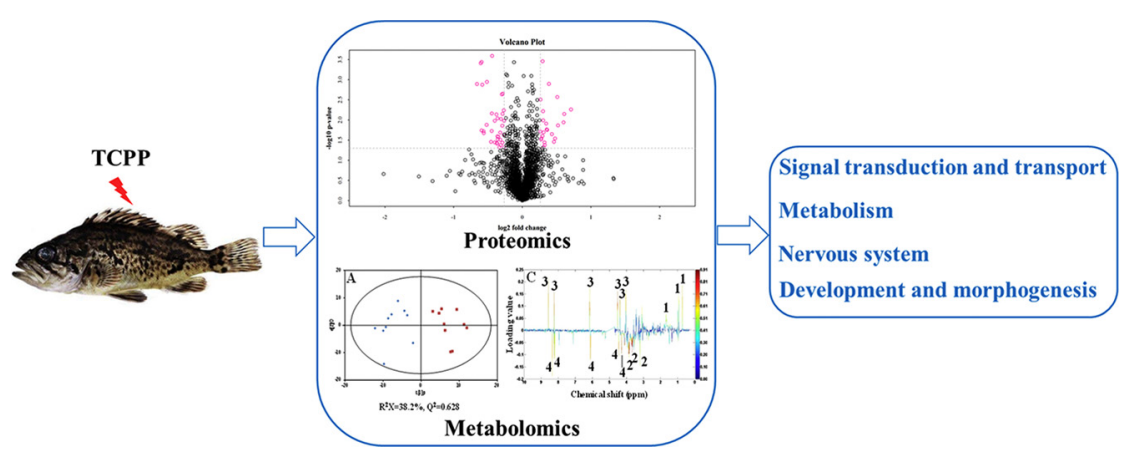

\begin{abstract}
A B S T R A C T
As alternatives of brominated flame retardants, organophosphate flame retardants (OPFRs) can be detected in multiple marine environmental media. Tris(1-chloro-2-propyl)phosphate (TCPP) was one of the most frequently and abundantly detected OPFRs in the Bohai Sea. Exposure to TCPP has been shown to induce abnormal behavior in zebrafish as well as neurotoxicity in Caenorhabditis elegans. However, there is a lack of mechanism investigations on the toxic effects of TCPP at molecular levels. In this work, proteomics and metabolomics were integrated to analyze the proteome and metabolome responses in rockfish Sebastes schlegeli treated with TCPP (10 and $100 \mathrm{nM}$ ) for 15 days. A total of 143 proteins and 8 metabolites were significantly altered in rockfish following TCPP treatments. The responsive proteins and metabolites were predominantly involved in neurotransmission, neurodevelopment, signal transduction, cellular transport, cholesterol metabolism, bile acid synthesis, and detoxification. Furthermore, a hypothesized network of proteins, metabolites, and pathways in rockfish was summarized based on the combination of proteomic and metabolomic results, showing some key molecular events in response to TCPP. Overall, the present study unraveled the molecular responses at protein and metabolite levels, which provided a better understanding of toxicological effects and mechanisms of TCPP in marine teleost.
\end{abstract}

(c) 2020 Elsevier B.V. All rights reserved.

\footnotetext{
* Corresponding author at: Center for Ocean Mega-Science, Chinese Academy of Sciences (CAS), Qingdao 266071, PR China.

E-mail address: hfwu@yic.ac.cn (H.Wu).
} 


\section{Introduction}

As alternatives of brominated flame retardants (BFRs), such as polybrominated diphenyl ethers (PBDEs), organophosphate flame retardants (OPFRs) have been widely produced and used in multiple fields, including electronic products, furniture and floor polish (van der Veen and de Boer, 2012). Because OPFRs are primarily presented as addictive rather than chemically bonded to materials, they are prone to be released to environmental media via volatilization, leaching and/or abrasion (van der Veen and de Boer, 2012). Therefore, OPFRs have been globally found in indoor and outdoor air (Li et al., 2012; Yang et al., 2014), water and sediment (Cao et al., 2012; Lee et al., 2018), and even animals and humans (Hou et al., 2016). OPFRs were also detected in seawaters and sediments from the Bohai Sea (Wang et al., 2015; Zhong et al., 2017), where the flame retardant industrial district of China is located. It is noteworthy that many OPFRs are already being marketed without proper assessments on their toxicological effects and environmental risk because of the urgent need for rapid BFRs replacement (Dishaw et al., 2011). Since OPFRs are structurally similar to organophosphate pesticides, a class of established neurotoxicants, increasing researches have revealed that OPFRs presented neurotoxic effects and developmental toxicity in animals (Abou-Donia et al., 2016; Dishaw et al., 2014; Du et al., 2019; Sun et al., 2016b; Yang et al., 2018). Considering the frequent detections of OPFRs in marine environment, it is necessary to concern the underlying influence of OPFRs on marine organisms, which has rarely been reported.

Around the Bohai Sea, tris(1-chloro-2-propyl)phosphate (TCPP) ranging from 4.6 to $921 \mathrm{ng} / \mathrm{L}$ in seawaters was one of the most frequently detected OPFRs (Wang et al., 2015; Zhong et al., 2017), which was similar to the observation that TCPP was the most frequently occurring OPFRs in marine fish and mussels from Swedish coastal areas (Sundkvist et al., 2010). Given its frequent detections in sea (Li et al., 2017), drinking water (Benotti et al., 2009), sewage effluent (Bollmann et al., 2012) and indoor air (Hartmann et al., 2004; Persson et al., 2018), TCPP has been classified by the U.S. EPA as a high hazard. Therefore, the underlying adverse effects of TCPP attract increasing attention. TCPP has been reported to show developmental and reproductive toxicity in pregnant rats (Environmental Protection Agency U.S., 2015), and induce abnormal swimming activity after exposure to TCPP ranging from $0.033-100 \mu \mathrm{M}$ for 5 days (Dishaw et al., 2014). Differing from other OPFRs, the neurotoxicity of TCPP was rarely reported. A previous study found that TCPP failed to induce locomotor deficits and dopaminergic degeneration in Caenorhabditis elegans until the exposure concentration as high as $500 \mathrm{mg} / \mathrm{L}$ (Xu et al., 2017). However, the molecular mechanisms of TCPP toxicity were still unknown.

Rockfish Sebastes schlegeli is a predatory species of seaperch and primarily distributes in northern Asia. In China, rockfish is an important economic species and can be found in the Bohai Sea and the Yellow Sea, where OPFRs were detected at high levels (Zhong et al., 2017). As far as we know, no study has been conducted to evaluate the toxic effects of TCPP in marine teleost, which would be suitable for evaluation of marine environmental risk of TCPP. Omics technologies based on high-throughput analysis have the ability to provide a global view of the cellular processes of organisms in response to environmental changes, such as pollutant stimulus, and hence uncover the toxicological mechanism of pollutant (Zhang et al., 2018). Especially, integrated omics approach that enables to provide more abundant information at transcript, protein, and metabolite levels has been frequently used in ecology and toxicity researches (Ji et al., 2019; Poulson-Ellestad et al., 2014; Sun et al., 2016a). To identify molecular events and pathways potentially involved in TCPP toxicities, proteomic and metabolomic profiles in rockfish treated with TCPP were evaluated by isobaric tags for relative and absolute quantification (iTRAQ) and nuclear magnetic resonance (NMR) techniques, respectively. The combination of protein and metabolite alterations presented a network of molecular responses in rockfish to TCPP, providing a better understanding of molecular mechanisms of TCPP toxicities on marine teleost.

\section{Materials and methods}

\subsection{Animals and TCPP treatments}

A hundred and twenty rockfish $S$. schlegeli (length: 5.5-6.0 cm, 5 months of age) were purchased from a local culturing farm (Yantai, China). After acclimated in aerated seawater in laboratory for 7 days, rockfish were randomly divided into three groups, including control, $10 \mathrm{nM}$ TCPP treatment, and $100 \mathrm{nM}$ TCPP treatment. The fish cultured in the normal filtered seawater containing $0.001 \%$ DMSO $(v / v)$ were used as solvent control group. Each treatment was implemented in two replicate tanks, each containing 20 individuals in $30 \mathrm{~L}$ of seawater. Seawater was daily replaced $2 / 3$ by fresh seawater. In order to theoretically maintain the designed exposure concentrations, fresh seawater was pre-added with DMSO for control group or TCPP for treated groups. During the acclimation and exposure periods, rockfish were kept in aerated water $\left(20 \pm 2{ }^{\circ} \mathrm{C}, 33 \mathrm{psu}\right)$ under a photoperiod of $12 \mathrm{~h}$ light and 12 dark, and fed with commercial bait daily. After exposure for 15 days, rockfish were immediately sampled and weighed. Then the whole fish were snap-frozen and ground into powder in liquid nitrogen for further analyses. Twelve rockfish (six from each replicate tank) were selected from each group for TCPP quantification, proteomics, and metabolomics analyses. To maintain results consistency, these analyses were implemented on rockfish from the same batch.

\subsection{Quantification of TCPP in rockfish samples}

The pretreatment and analytical method for the determination of TCPP referred to description in a previous publication (Zhong et al., 2017). The dried rockfish powder $(0.35 \pm 0.02 \mathrm{~g}, n=12)$ were spiked with $40 \mathrm{ng}$ of $\mathrm{d}_{12}$-Tris(2-carboxyethyl)phosphine (TCEP) to determine recovery of the internal standard, followed by being subjected to Soxhlet extraction for $24 \mathrm{~h}$ in $200 \mathrm{~mL}$ of dichloromethane at $52{ }^{\circ} \mathrm{C}$. The extracts were evaporated to $10 \mathrm{~mL}$ using hexane as the keeper. A volume of $1 \mathrm{~mL}$ of the concentrated extracts were taken out for fat weight determination and the remaining $9 \mathrm{~mL}$ of the extracts were further concentrated to about $2 \mathrm{~mL}$ in methanol under gentle nitrogen flows in Teflon tubes. After frozen at $-20{ }^{\circ} \mathrm{C}$ overnight, the extracts $(2 \mathrm{~mL})$ were centrifuged and the supernatants were transferred into separating funnels, diluted by $500 \mathrm{~mL}$ of ultra-pure water and subjected to solid phase extraction by gravity with HLB (hydrophilic-lipophilic balance) columns $\left(6 \mathrm{~cm}^{3}, 200 \mathrm{mg}\right)$. Then, the columns were drained and eluted with $10 \mathrm{~mL}$ of ethyl acetate. The elutes were frozen at $-20^{\circ} \mathrm{C}$ for $24 \mathrm{~h}$ to remove water and the residual water was removed again by adding baked $\mathrm{Na}_{2} \mathrm{SO}_{4}$. The dewatered eluates were evaporated to around $200 \mu \mathrm{L}$ under gentle nitrogen flows and transferred to new vials. Finally, 20 ng of ${ }^{13} \mathrm{C}_{6}$-polychlorinated biphenyl 208 (PCB 208) was added as an injection standard before instrumental analysis.

Tissue extracts were analyzed using gas chromatography-mass spectrometry (GC-MS) on a gas chromatograph (Agilent 7890A) coupled to a mass spectrometer (Agilent 5975C). The gas chromatograph was equipped with a DB-5MS column $(30 \mathrm{~m} \times 0.25 \mathrm{~mm} \times 0.25 \mu \mathrm{m})$ and the heating grogram was as follows: $80{ }^{\circ} \mathrm{C}(3 \mathrm{~min}) \rightarrow 8^{\circ} \mathrm{C}$ min$1 \rightarrow 150{ }^{\circ} \mathrm{C}(0 \mathrm{~min}) \rightarrow 5{ }^{\circ} \mathrm{C} \min -1 \rightarrow 300{ }^{\circ} \mathrm{C}(5 \mathrm{~min})$. The quadrupole was set at $150{ }^{\circ} \mathrm{C}$ and the ion source of the mass spectrometer was maintained at $230{ }^{\circ} \mathrm{C}$ and $70 \mathrm{eV}$ electron impact. Calibration curves derived from eight gradients $\left(0,10,20,50,100,200,500\right.$, and $\left.1000 \mathrm{pg} \mathrm{L}^{-1}\right)$ were used to quantify the compounds of focus. The method of blank and recovery for TCPP was $0.0025 \pm 0.00014 \mathrm{nM}$ and $88.99 \pm 15.22 \%$ ( $n=$ 3 ). All the data related to TCPP concentrations in rockfish were corrected by the blank and recovery processed and analyzed by IBM SPSS statistics 22. Moreover, the theoretical bioconcentration factor 
(BCF) could be calculated by the concentration of TCPP in rockfish divided by the theoretical concentration of TCPP in exposure water.

\section{3. iTRAQ-based proteomic analysis}

Rockfish samples were dissolved in lysis buffer ( 9 M urea, 4\% CHAPS, $1 \%$ w $/ v$ DTT) with protease inhibitor (Roche Applied Science, Mannheim, Germany). The concentrations of the protein extracts were determined using the Bradford method (Bradford, 1976). iTRAQ reagent was applied for quantitative proteomic analysis according to the manufacturer's instructions (Applied Biosystems). Briefly, $100 \mu \mathrm{g}$ of protein extracted from each treatment was dissolved in the dissolution buffer (AB SCIEX, MA, USA). After being reduced, alkylated and trypsindigested, the protein samples were then labeled using iTRAQ 8-plex reagents (AB SCIEX, MA, USA). Control group (labeled with 113- and 114tags) were in duplicate, while $10 \mathrm{nM}$ TCPP treatment (labeled with 115, 116- and 117- tags) and $100 \mathrm{nM}$ TCPP treatment (labeled with 118-, 119- and 121- tags) were in triplicate. In control group, 6 rockfish samples that were equally taken from two tanks were pooled as one replicate. In 10 and $100 \mathrm{nM}$ TCPP-treated groups, 4 rockfish samples that were equally taken from two ranks were pooled as one replicate. iTRAQ labeled peptides were firstly fractionated by strong cation exchange (SCX) chromatography using the AKTA Purifier system (GE Healthcare). Then each fraction was injected for HPLC analysis, followed by LC-MS/MS analysis performed on a Q Exactive mass spectrometer (Thermo Scientific) that was coupled to Easy nLC (Thermo Fisher Scientific).

MS/MS spectra were searched using MASCOT engine (Matrix Science, London, UK) embedded into Proteome Discover 1.4 against a perciformes protein database (uniprot_Perciformes_58748_20180316. fasta). The database search parameters were the followings: iTRAQ 8plex quantification; enzyme: trypsin digestion; max missed cleavages: 2 ; variable modifications: oxidation; peptide mass tolerance: \pm 20 ppm; fragment mass tolerance: $0.1 \mathrm{Da}$; peptide FDR: $\leq 0.01$. The protein ratios were calculated as the median of only unique peptides of the protein, and only those proteins with fold change ratios $>1.2$ or $<0.83$ as well as a $p$ value $<.05$ were considered as differentially expressed proteins (DEPs).

The protein sequences of DEPs were locally searched against SwissProt database (mouse) using the NCBI BLAST + client software to find homologue sequences from which the functional annotation could be transferred to the studied sequences. Then the homologue sequences were loaded into Blast2GO (Version 3.3.5) for Gene Ontology (GO) mapping and annotation, and blasted against the online Kyoto Encyclopedia of Genes and Genomes (KEGG) database (http:// geneontology.org) for KEGG pathway annotation. GO annotation included three ontologies (biological process, BP; molecular function, MF; cellular component, $\mathrm{CC}$ ). To further explore the impact of DEPs in cell physiological process and discover internal relations between DEPs, enrichment analysis was conducted. GO enrichment and KEGG pathway enrichment analyses were applied based on Fisher's exact test. Benjamini-Hochberg for multiple testing was further used to adjust derived $p$ value. And only functional categories and pathways with $p<.05$ were considered as significant.

\subsection{Nuclear magnetic resonance-based metabolomic analysis}

NMR-based metabolomics was used to unravel the alterations of metabolites in rockfish treated with TCPP. Polar metabolites in rockfish ( $n=10$ for each treatment) were extracted in $4 \mathrm{~mL} / \mathrm{g}$ of methanol, $5.25 \mathrm{~mL} / \mathrm{g}$ of water and $2 \mathrm{~mL} / \mathrm{g}$ of chloroform as described previously and analyzed on a Bruker AV 500 NMR spectrometer performed at $500.18 \mathrm{MHz}$ (at $25^{\circ} \mathrm{C}$ ) as described previously (Ji et al., 2015). All one dimensional ${ }^{1} \mathrm{H}$ NMR spectra were converted to a data matrix using the custom-written ProMetab software in Matlab version 7.0 (The MathsWorks, Natick, MA, USA) and then segmented into bins with a width of $0.005 \mathrm{ppm}$ between 0.2 and $10.0 \mathrm{ppm}$. All the NMR spectra were generalized log transformed with a transformation parameter $\lambda=1.0 \times 10^{-8}$ and subsequently data were mean-centered before data analysis. The supervised multivariate data analysis methods, partial least squares discriminant analysis (PLS-DA) and orthogonal projection to latent structure with discriminant analysis (O-PLS-DA), were sequentially carried out to uncover and extract the statistically significant metabolite variations related to TCPP treatments. Metabolites were identified by using the software, Chenomx (Evaluation Version, Chenomx Inc., Edmonton, Alberta, Canada). More details were available in Supplementary Material 1.

\section{Results and discussion}

\subsection{TCPP accumulation in rockfish}

After exposure with TCPP for 15 days, significant accumulations of TCPP were observed in rockfish from both TCPP treatments. By contrast, the concentration of TCPP in rockfish from control group was below the detection limit ( $0.76 \mathrm{pM})$. In details, rockfish from 10 and $100 \mathrm{nM}$ TCPPtreated groups accumulated $423.94 \pm 69.13$ and $1510.46 \pm 165.78 \mathrm{ng} / \mathrm{g}$ TCPP (lipid weight, LW), respectively. The theoretical values of BCF of TCPP were 2.98 and $0.97 \mathrm{~L} / \mathrm{Kg}$ (wet weight, WW) in 10 and $100 \mathrm{nM}$ TCPP-treated rockfish, respectively, which were lower than those in sea urchin, ranging from 19 to $41 \mathrm{~L} / \mathrm{Kg}$ WW (Campoy-López et al., 2018). All these BCFs of TCPP were far lower than the threshold $(2000 \mathrm{~L} / \mathrm{kg}$ ) under REACH by European Union, indicating that TCPP cannot be regarded as bioconcentrable. Nevertheless, several molecular responses such as metabolites and proteins were significantly altered in rockfish as a result of TCPP treatments.

\subsection{Overview of proteomic and metabolomic responses}

Utilization of iTRAQ-based proteomic analysis on rockfish whole body led to identification of 2295 proteins, among which 143 proteins were significantly altered (fold change $>1.2$ or $<0.83, p<.05$ ) as a result of TCPP treatments. A total of 61 and 120 proteins were significantly altered in 10 and $100 \mathrm{nM}$ TCPP-treated groups, respectively (Fig. 1, Supplementary Material 2). Among these proteins, 38 proteins (26.57\%) were commonly altered in both TCPP treatments. Furthermore, TCPP induced more inhibition than promotion in rockfish at protein levels. In details, 24 up- and 37 down-regulated proteins were identified in $10 \mathrm{nM}$ TCPP-treated group, while $100 \mathrm{nM}$ TCPP treatment induced 40 up- and 80 down-regulated proteins (Fig. 1).

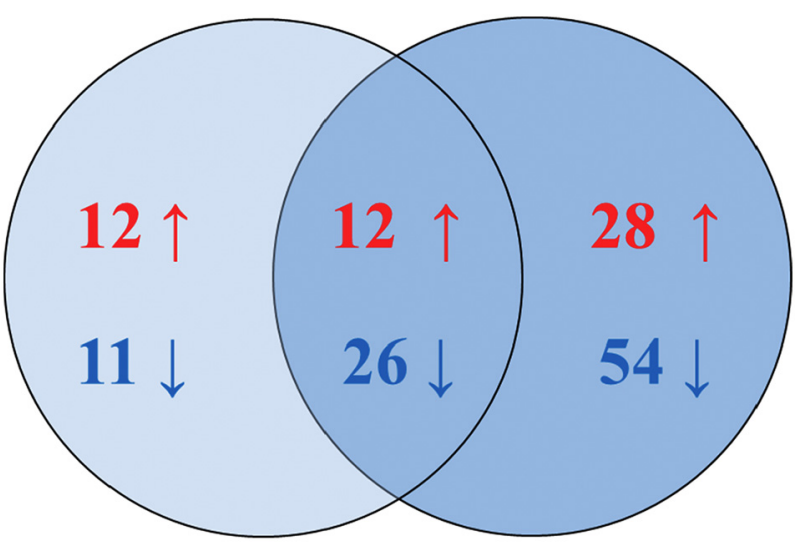

$10 \mathrm{nM}$ TCPP

$100 \mathrm{nM}$ TCPP

Fig. 1. A Venn diagram shows the number of proteins differentially expressed in 10 and $100 \mathrm{nM}$ TCPP groups. $\uparrow$ and $\downarrow$ represent up- and down-regulation, respectively. 
Due to few GO terms and KEGG pathways enriched, a relax criterion that based on $p$ value, not adjusted $p$ value, for enrichment selection was considered in this work. GO enrichment analysis revealed that 51 and 38 GO terms were significantly over enriched in 10 and 100 nM TCPPtreated groups, respectively (Supplementary Table). The 20 most enriched GO terms were listed in Fig. 2. KEGG pathway enrichment analysis revealed that 5 and 22 KEGG pathways were over enriched in 10 and $100 \mathrm{nM}$ TCPP-treated groups, respectively (Fig. 3). Though more than a quarter of DEPs (26.57\%) were commonly identified in both TCPP-treated groups with same expression tendency, the enriched pathways totally differed from each other, which might result from each DEP being involved in multiple pathways.

NMR-based metabolomics was performed on the rockfish whole body to characterize the alteration of metabolite profiles in response to TCPP treatment. O-PLS-DA demonstrated the significant $(p<.05)$ metabolic differences between rockfish samples from control group and TCPP-treated groups with reliable $Q^{2}$ values of 0.628 and 0.435 (Fig. 4). As shown in Figs. 4, $10 \mathrm{nM}$ TCPP-treated rockfish had significantly $(p<.05)$ higher levels of cholate and AMP, and lower levels of sn-glycero-3-phosphocholine (GPC) and inosine than those in control group. In $100 \mathrm{nM}$ TCPP-treated rockfish, cholate and ethanol were elevated, whereas glutamate, glutamine, and aspartate were decreased. The low (10 nM) and high (100 nM) concentrations of TCPP induced different metabolomic profiles except for the commonly increased cholate.

Combination of metabolomics and proteomics analysis enables to provide a better understanding of molecular events in response to TCPP. Based on the altered proteins and metabolites, hypothesized networks in rockfish in response to TCPP were thus summarized. Overall, multiple biological pathways including neurodevelopment and neurotransmission, signaling transduction, transport, metabolism, and detoxification (Fig. 5) were significantly influenced at molecular level as a result of TCPP treatments.

\subsection{Neurodevelopment and neurotransmission}

Previous studies showed the neurotoxicity induced by OPFRs in cells, fishes and rats, including influences in neurodevelopment and neurotransmission (Abou-Donia et al., 2016; Gu et al., 2018; Slotkin et al., 2017; Sun et al., 2016b; Yang et al., 2018). These studies focused on the changes of known endpoints like acetylcholinesterase (AChE), which was difficult to characterize the molecular mechanism of neurodevelopment and neurotransmission dysregulation.

In this work, several proteins involved in neurotransmitter secretion, regulation of neurotransmitter levels, signal release, synaptic signaling, and cholinergic synapse were significantly affected by TCPP treatments. Synapsins has been proposed to be involved in regulation of neurotransmitter release at synapses, and act as a link between extracellular stimuli and the intracellular signaling events and could be regulated by several protein kinases and phosphatases (Cesca et al., 2010). It has been reported that synapsin I is implicated in presynaptic neurotransmission, whereas synapisn II has little involvement in kindling (Morimoto et al., 1998). Synapsin I mutant mice showed marked decrease of glutamate release from nerve endings (Li et al., 1995). In this work, synapsin I was also down-regulated in 100 nM TCPPtreated rockfish, suggesting that neurotransmission associated with synapsin I was impeded in pre-synaptic neuron. As the most abundant amino acid neurotransmitter in brain (Zhou and Danbolt, 2014), glutamate decrease was also identified in rockfish treated with $100 \mathrm{nM} \mathrm{TCPP.}$ Unfortunately, the decrease of glutamate in whole body failed to reflect the alteration of glutamate functioning as neurotransmitter. $\alpha$-amino3-hydroxy-5-methyl-4-isoxazolepropionic acid receptors (AMPARs) are cation-permeable ionotropic glutamate receptors, and are abundant and widely distributed in post-synaptic of central nervous system (Beneyto and Meador-Woodruff, 2003). AMPARs are highly dynamic whose change in number is one of the major ways by which the efficacy of neurotransmission can be altered (Chater and Goda, 2014). The upregulated AMPAR suggested that neurotransmission in post-synaptic neuron was enhanced. As a neuronal calcium sensor, neurocalcin delta (ND) has been reported to be implicated in the regulation of endocytosis-dependent neuronal functions such as axonal growth and branching (Yamatani et al., 2010). Neural cell adhesion molecule (NCAM) mediates neuronal functions by controlling calciumindependent intercellular adhesion, neurite outgrowth, and cell migration (Francavilla et al., 2007). The activation of NCAM has been frequently reported to be associated with increase of intracellular $\mathrm{Ca}^{2+}$ (Sheng et al., 2013). In this work, NCAM and ND were commonly upregulated in both TCPP treatments, suggesting $\mathrm{Ca}^{2+}$ influx into synaptic neuron, which might be attributed to AMPAR up-regulation (Fig. 5A). AMPAR, ND, NCAM, and synapsins were almost uniquely expressed in nervous system, whose contents in whole body therefore could reflect the influence of TCPP on neurotransmission in rockfish. Hereby, the
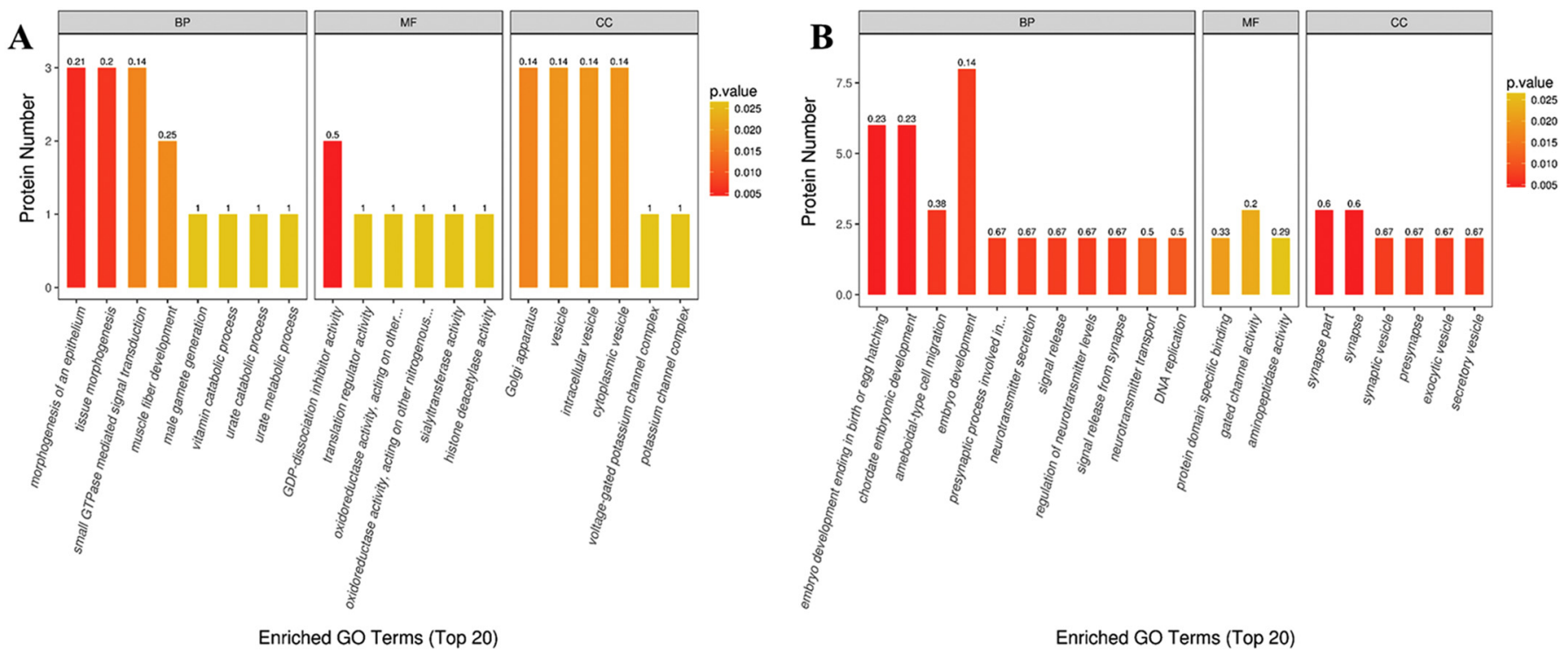

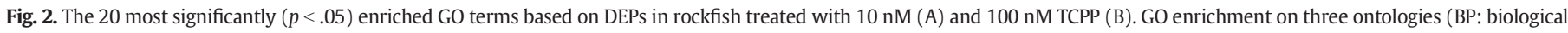

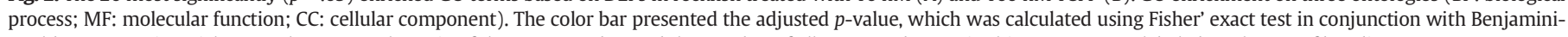
Hochberg correction. richFactor that means the ratio of the DEPs number and the number of all annotated genes in this GO term was labeled on the top of bar diagram. 

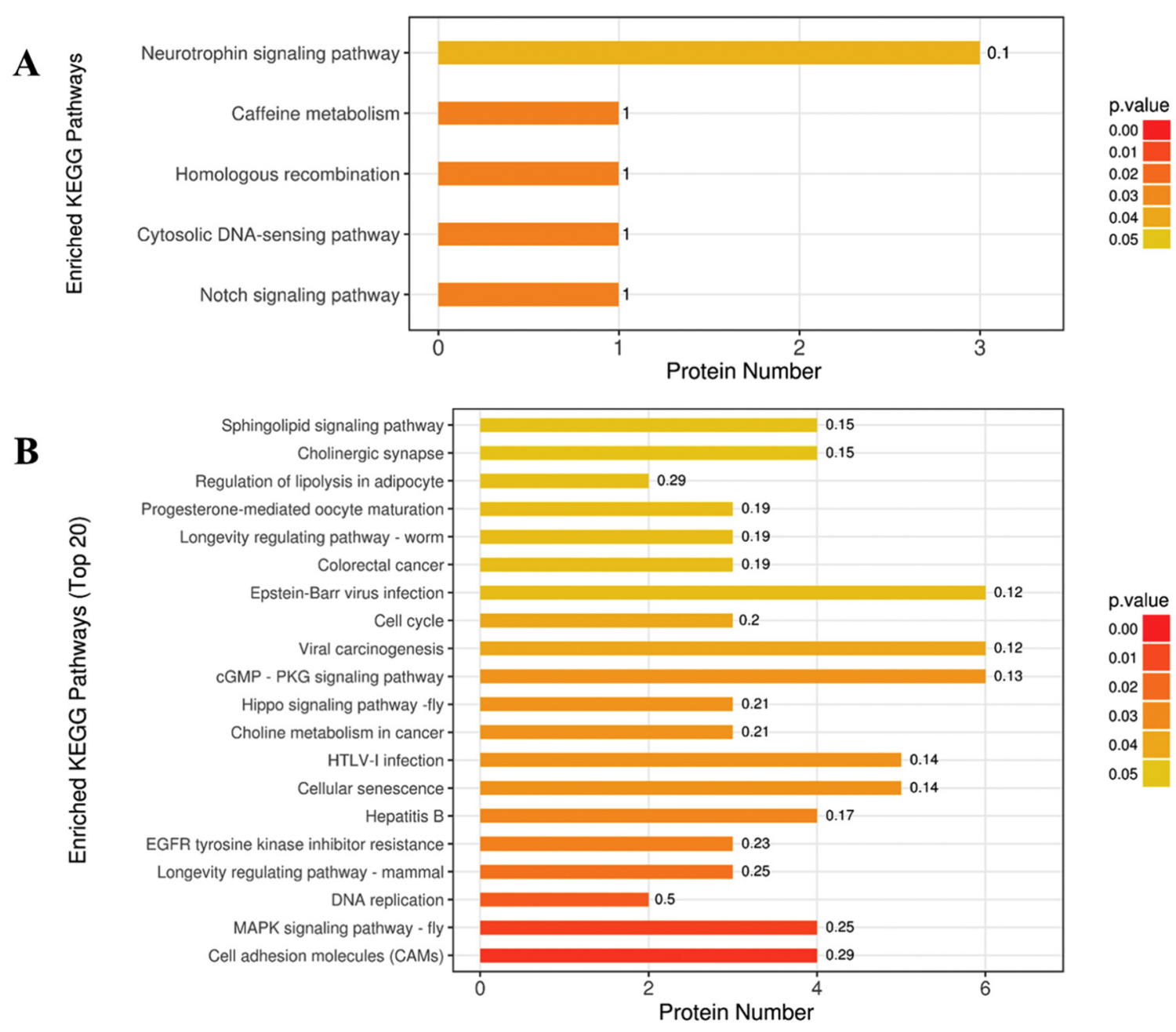

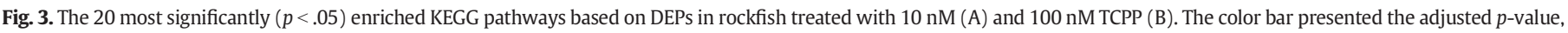

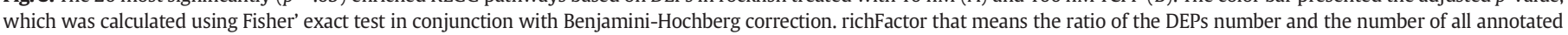
genes in this KEGG pathway was labeled on the top of bar diagram.

down-regulated synapsin I as well as the up-regulated AMPAR, NCAM, and ND in whole body of rockfish suggested the dysregulation of neurotransmission induced by TCPP, which might subsequently elicit disorders of neurodevelopment.

As expected, several proteins that were associated with neurodevelopment were significantly altered in response to TCPP treatment. Phosphatidylinositol-binding clathrin assembly protein (PBCAP) and dihydropyrismidinase-like 9 (DPYSL9) were down-regulated in both TCPP-treated groups. Similar to neurocalcin, PBCAP plays a key role in clathrin-mediated endocytosis which is important in processes such as internalization of cell receptors and synaptic transmission, and was reported to be associated with Alzheimer's disease (Harold et al., 2009). DPYSL9 have a function of neuronal differentiation and/or axon growth. Similarly, the decrease of dihydropyrimidinase was also observed in Caenorhabditis elegans treated with organophosphorus pesticides (Lewis et al., 2009). Furthermore, organophosphorus compounds might exert its toxicity via regulation of neuronal cytoskeleton (AbouDonia, 1993; Flaskos, 2014), which was consistent with the downregulation of cytoskeletal protein like neurofilament in $100 \mathrm{nM}$ TCPPtreated rockfish. These proteins including PBCAP, DPYSL9, and neurofilament were suppressed as a result of TCPP treatment, suggesting that TCPP might impede the neurodevelopment like axonal growth and branching. Differing from the previous reports that AChE (Abou-Donia et al., 2016; Sun et al., 2016b; Yang et al., 2018) or O-linked $N$ acetylglucosamine transferase (Gu et al., 2018) were the key targets of OPFRs, we identified many novel proteins related to neurotransmission and neurodevelopment in response to TCPP, which could be selected as the potential biomarkers (Fig. 5A).

\subsection{Signal transduction and cellular transport}

Signal transduction is the transmission of molecular signals from a cell's exterior to its interior, which is initiated by cell-surface receptors. Signal transduction is prone to be targeted by contaminants because of its similarity to ligands, followed by a series of intracellular responses (Costa, 1998). In this work, the signal transduction pathways related proteins, such as Rho proteins and cytoskeletal proteins, were significantly affected by TCPP. As it is known, Rho GTPases related proteins together with cytoskeletal proteins were at the crossroad of several signaling network (Hervé and Bourmeyster, 2015). Thus, the alterations of Rho proteins and cytoskeletal proteins suggested influences of TCPP treatments on signal pathways, which could be supported by the results of KEGG pathway enrichment analysis.

KEGG pathway enrichment analysis (Fig. 3) showed that a total of 7 signaling pathways were significantly enriched in TCPP-treated groups. In $10 \mathrm{nM}$ TCPP-treated group, neurotrophin signaling pathway were significantly enriched, indicated by differential expressions of Rhorelated GTP-binding protein ( $\mathrm{RrGbP}$ ), cell division cycle 42-like protein (CDC42IP), and Rho GDP-dissociation inhibitor 1 (RGdI1). Neurotrophin signaling pathway controls many aspects of survival, development and function of neurons (Reichardt, 2006). Both RrGbP and CDC42lP belong to GTPase family and cycle between active GTP-bound and an inactive 

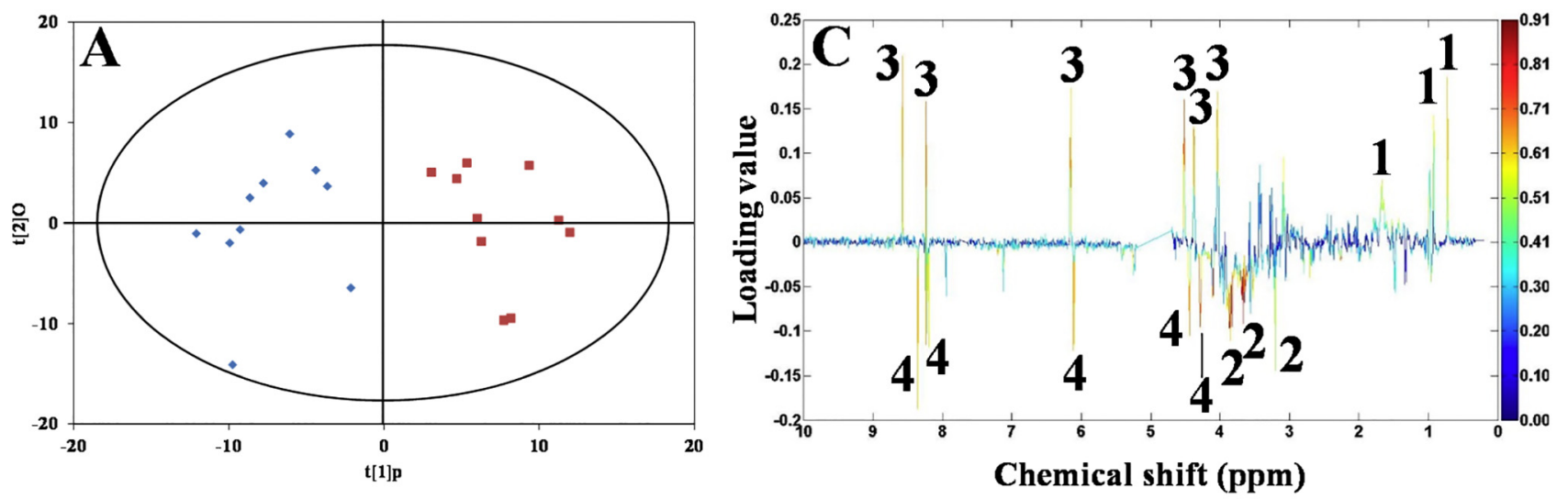

$R^{2} X=38.2 \%, Q^{2}=0.628$
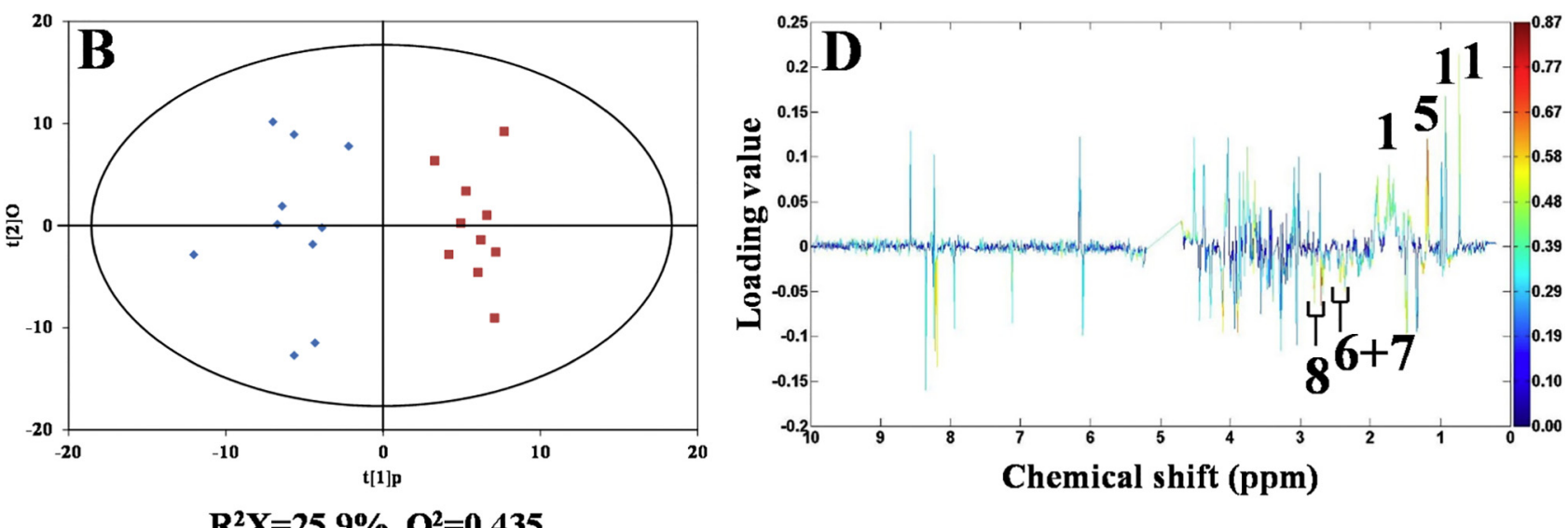

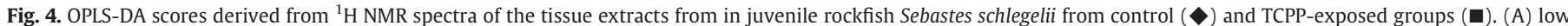

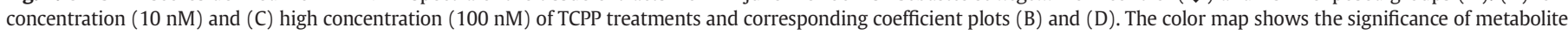

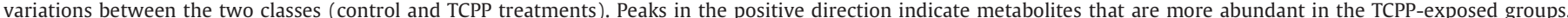

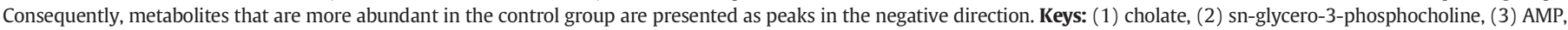
(4) inosine, (5) ethanol, (6) glutamate, (7) glutamine and (8) aspartate.

GDP-bound state. RGdI1 can control Rho proteins homeostasis by inhibiting the dissociation of GDP from them and the subsequent binding of GTP to them (Zhang et al., 2005). Therefore, up-regulated RrGbP and CDC42IP and down-regulated RGdI1 suggested that $10 \mathrm{nM}$ TCPP treatment enhanced neurotrophin signaling pathway in rockfish. In 100 nM TCPP treatment group, MAPK signaling pathway, Hippo signaling pathway, cGMP-PKG signaling pathway, and sphingolipid signaling pathway were significantly enriched. In particular, the enhancement of cGMP-PKG signaling pathway was markedly elicited by $100 \mathrm{nM}$ TCPP treatment, which was indicated by common up-regulations of solute carrier (SLC) family 25 member 4 (SLC25A4), RrGbP, peptidyl-prolyl cis-trans isomerase, voltage dependent anion channel protein (VDAC), guanine nucleotide binding protein ( $G$ protein), alpha inhibiting activity polypeptide, and $\mathrm{V}$-akt murine thymoma viral oncogene homologue 2. cGMP-PKG predominantly mediates the action of nitric oxide and natriuretic peptides and regulates a broad array of physiological processes including synaptic plasticity (Ren et al., 2014). The promoted of cGMP-PKG signaling pathway maybe contribute to the dysregulation of neurodevelopment.

As the second largest family of membrane proteins after $G$ proteincoupled receptors, SLC superfamily of transporters functions as regulating ion fluxes at the plasma membranes, or solute transport into and out of cellular organelles (Cesar-Razquin et al., 2015). A newly published work showed that organophosphorus pesticides could inhibit activities of SLC transporters (SLC22 and SLC47) in human HEK293 cells (Chedik et al., 2019). Similarly, SLC25, SLC35, and SLC44, were altered in TCPP- treated rockfish in this work. SLC25 member 4 (SLC25A4) is also known as adenine nucleotide translocator (ANT) and serves as mitochondrial ADP/ATP transporter (Porcelli et al., 2014), which was significantly up-regulated in both TCPP-treated groups. Furthermore, voltage-dependent anion-selective channel (VDAC) that locates in the outer mitochondrial membrane and plasma membrane functions as transporting ATP, $\mathrm{Ca}^{2+}$, and other metabolites. VDAC has been reported to be relevant to ATP rationing, $\mathrm{Ca}^{2+}$ homeostasis, protection against oxidative stress, regulation of apoptosis (Shoshan-Barmatza et al., 2010). The common up-regulations of ANT and VDAC1 may cause depolarization and $\Delta \Psi$ collapse in mitochondria. In addition, SLC44A2 mediates choline transport across the plasma and mitochondrial membranes (Inazu, 2014). The significant down-regulation of SLC44A2 in both TCPP treatments suggested that TCPP might impede choline transport. Though choline was not altered in TCPP-treated rockfish, GPC that functions as one of two major forms of choline storage was significantly decreased in $10 \mathrm{nM}$ TCPP-treated rockfish, indicating that TCPP treatment indeed suppressed choline influx (Fig. 5B). The decrease of choline storage might subsequently disturb acetylcholine synthesis and affect neurotransmission.

\subsection{Metabolism}

Metabolism produces energy and key intermediates for multiple life processes, playing a critical role in resistance to environmental changes and xenobiotic stimulus (Dong and Zhang, 2016; Sokolova, 2013). As a 

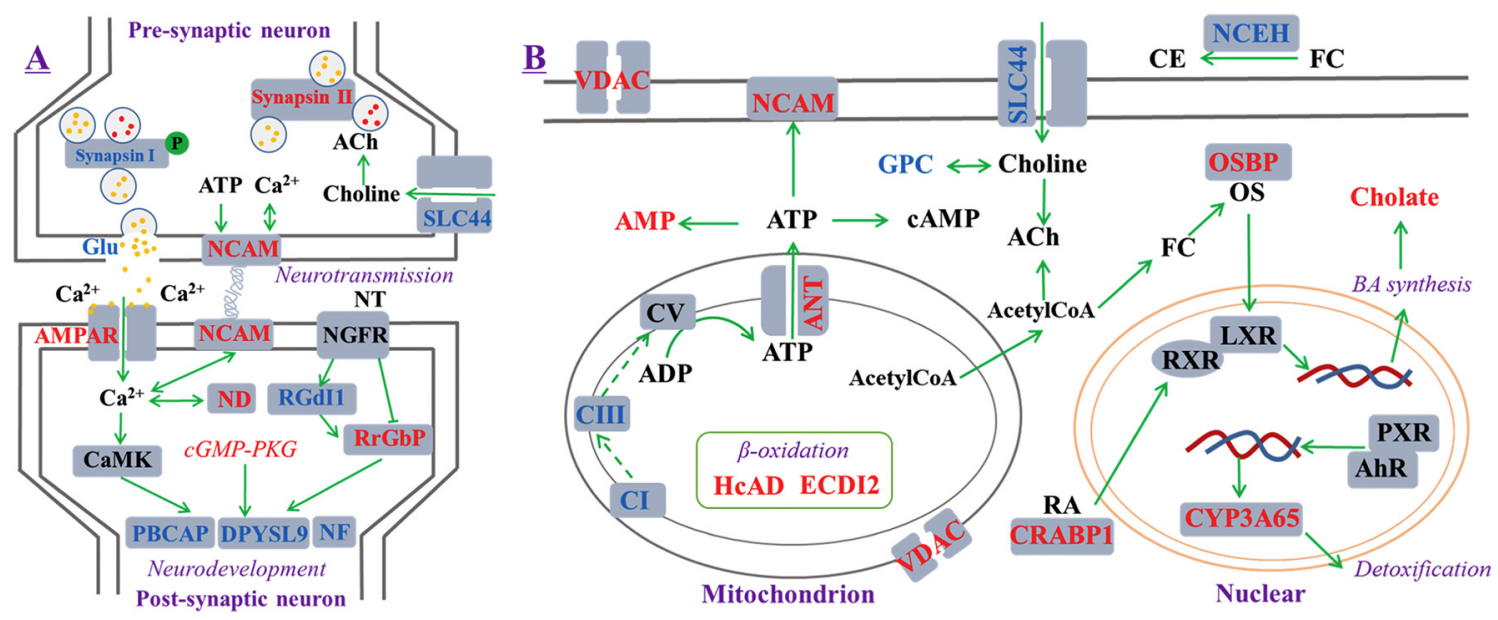

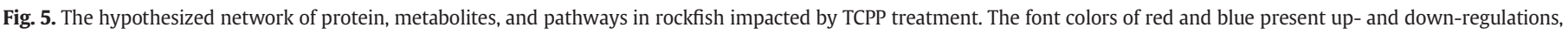

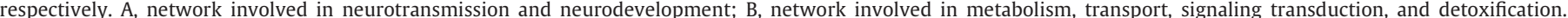

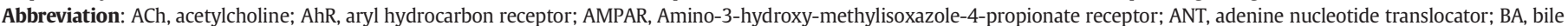

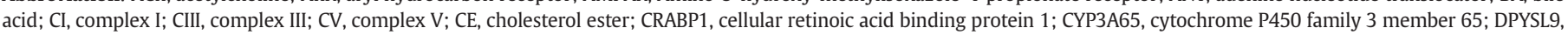

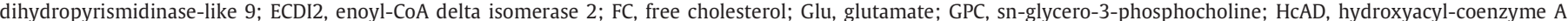

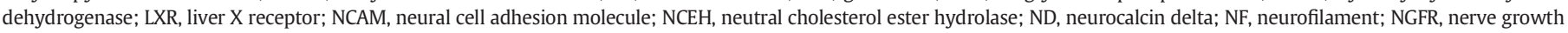

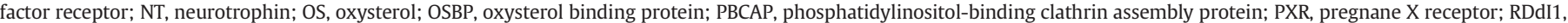
Rho GDP-dissociation inhibitor 1; RrGbP, Rho-related GTP-binding protein; RXR, retinoid X receptor; SLC44, solute carrier family 44; VDAC, voltage dependent anion channel protein.

matter of fact, a previous study revealed that another typical OPFR, triphenyl phosphate (TPP), could induce adverse effects on metabolism as well, such as disruption in carbohydrate metabolism and lipid metabolism (Du et al., 2016).

In this work, numerous DEPs and metabolic pathways associated with metabolism were significantly changed as a result of TCPP treatment. Cholesterol serves as an important precursor for the biosynthesis of steroid hormones, bile acid and vitamin D. Neutral cholesterol ester hydrolase 1 (NCEH) plays a vital role in hydrolysis of intracellular cholesterol ester (CE) and removing cholesterol from macrophages (Ross et al., 2014). In fact, previous studies revealed that $>50$ serine hydrolases and receptors, including NCEH, were the targets of organophosphorus toxicants (Casida and Quistad, 2005; Sakai et al., 2014). In this work, NCEH was down-regulated in $100 \mathrm{nM}$ TCPP-treated rockfish, suggesting that TCPP could disturb cholesterol homeostasis by inhibiting NCEH in marine teleost. Oxysterol is well known as a molecular regulator of cholesterol metabolism and transport, and bile acid synthesis by binding liver X receptor $\alpha$ (LXR $\alpha)$ (Olkkonen et al., 2012). Oxysterol binding protein (OSBP) functioning as an oxysterol ligand with receptor has been reported to in part control oxysterol by complex interaction (Schroepfer, 2000). The up-regulated OSBP in TCPP-treated rockfish suggested that the cholesterol homeostasis might be disturbed and bile acids synthesis might be enhanced via regulating oxysterol (Fig. 5B). Moreover, metabolomic analysis showed that cholate was significantly increased in TCPP-treated rockfish (Fig. 4). As the major component of primary bile acids, cholate elevation was in agreement with the hypothesized promoted bile acid synthesis and irregular cholesterol homeostasis (Fig. 5B).

Mitochondria play key roles in ATP production, apoptosis, calcium cycle, fatty acid metabolism, amino acid metabolism, and heme biosynthesis, which have been frequently reported as the target of contaminant-induced toxicity (Ji et al., 2019; Meyer et al., 2013; Sokolova et al., 2005). As the complex I and III of oxidative phosphorylation, NADH dehydrogenase and cytochrome $c$ reductase are vital in electron transporting. In this work, marked down-regulations of NADH dehydrogenase and cytochrome $c$ reductase were induced in 100 nM TCPP-treated group, which might be associated with the upregulated ANT and VDAC. It is noteworthy that the disruption of energy generation might subsequently induce other dysregulation of biological processes. A previous study revealed that decrease of ATP levels induced the up-regulation of NCAM, which plays key a role in neurodevelopment (Nagao et al., 2010). Moreover, both hydroxyacylcoenzyme A dehydrogenase (HcAD) and enoyl-CoA delta isomerase 2 (EcDI2) are associated with $\beta$-oxidation (Palosaari et al., 1990; Schulz et al., 2011). The increased expressions of HcAD and EcDI2 suggested that $\beta$-oxidation was enhanced.

GO enrichment analysis (Fig. 2) indicated that a variety of metabolic processes, including urate catabolic and metabolism processes, isoprenoid catabolic processes, vitamin catabolic processes, were significantly enriched in $10 \mathrm{nM}$ TCPP-treated group, which was attributed to the alteration of cellular retinoic acid binding protein 1 (CRABP1). CRABP1 is assumed to play an important role in retinoid-mediated differentiation and proliferation processes by binding to retinoid acid and helping to transport it into cells. The retinoid transport, metabolism and signaling were frequently found to be disrupted by environmental pollutants, such as pesticides, polychlorinated dioxins, polychlorinated biphenyls, and polycyclic aromatic compounds (Novak et al., 2008). In this work, down-regulation of CRABP1 in $10 \mathrm{nM}$ TCPP-treated group signified that TCPP at low concentration could affect retinoic acid-mediated biological processes.

\subsection{Detoxification}

Cytochromes P450 (CYPs) are a superfamily of enzymes that are important for clearance of various substances and hormone synthesis and breakdown (Danielson, 2002). In this work, two cytochrome P450 proteins (CYP2U1 and CYP3A65) were identified to increase in response to 100 nM TCPP treatment. Both CYP2 and CYP3 were involved in xenobiotic metabolism and can be induced by their metabolic substrates through the action of nuclear receptor transcription factors. In particular, CYP3A65 was firstly identified in zebrafish and its transcription required pregnane $X$ receptor (PXR), aryl hydrocarbon receptor (AhR) 2 DNA and ligand-binding domains (Chang et al., 2013). The alteration of CYP3A65 in this work was analogous with previous studies, revealing that its analogue CYP3A37 was induced in chicken and hepatocytes cells in response to TCPP (Crump et al., 2012; Farhat et al., 2013). Overall, the responses of CYP2U1 and CYP3A65 suggested that these two CYPs were involved in detoxification of TCPP in rockfish. 


\section{Conclusions}

In this work, we measured the bioaccumulations of TCPP in juvenile rockfish Sebastes schlegeli and evaluated the proteome and metabolome responses. Though significant accumulations of TCPP were observed in rockfish treated with TCPP (10 and $100 \mathrm{nM}$ ) for 15 days, TCPP still could not be considered as bioconcentrable in rockfish because of its poor BCF. iTRAQ-based proteomic analysis demonstrated that TCPP treatments resulted in significant alterations of 143 proteins, which were mainly associated with neurotransmission, neurodevelopment, signal transduction, transport, metabolism, detoxification. The complementary use of NMR-based metabolomics identified 8 changed metabolites in response to TCPP treatment. Based on the responsive proteins, metabolites, and pathways, the hypothesized network in rockfish impacted by exposure to TCPP were summarized (Fig. 5). Though the present study showed that TCPP treatments induced significant alterations of multiple proteins and metabolites, further studies focusing on targeted organs like brains and livers as well as behavioral and biochemical endpoints were suggested to provide complementary information on TCPP toxicity. Overall, a combination of metabolomics and proteomics analysis showed the capability of identifying molecular events in response to TCPP, which would contribute to better understanding toxicological mechanisms of TCPP.

\section{CRediT authorship contribution statement}

Chenglong Ji:Conceptualization, Writing - original draft, Investigation.Zhen Lu:Investigation.Lanlan Xu:Investigation.Fei Li:Resources, Writing - review \& editing.Ming Cong:Validation.Xiujuan Shan:Resources, Validation.Huifeng Wu:Supervision, Writing - review \& editing.

\section{Declaration of competing interest}

The authors declare that they have no known competing financial interests or personal relationships that could have appeared to influence the work reported in this paper.

\section{Acknowledgement}

This research was supported by the grants from National Natural Science Foundation of China (41976152, 21677173), Yantai Institute of Coastal Zone Research, Chinese Academy of Sciences (YICY855011023), Youth Talent Program Supported by Laboratory for Marine Fisheries Science and Food Production Processes, Pilot National Laboratory for Marine Science and Technology (Qingdao) (No. 2018-MFST02) and the Youth Innovation Promotion Association, Chinese Academy of Sciences (2017255).

\section{Appendix A. Supplementary data}

Supplementary data to this article can be found online at https://doi. org/10.1016/j.scitotenv.2020.138307.

\section{References}

Abou-Donia, M.B., 1993. The cytoskeleton as a target for organophosphorus ester-induced delayed neurotoxicity (opidn). Chem. Biol. Interact. 87, 383-393.

Abou-Donia, M.B., Elgamal, M., Elkholi, I., Wang, Q., 2016. Organophosphorus flame retardants (OPFR): neurotoxicity. J. Environ. Health Sci. 2, 1-29.

Beneyto, M., Meador-Woodruff, J.H., 2003. Expression of transcripts encoding AMPA receptor subunits and associated postsynaptic proteins in the macaque brain. J. Comp. Neurol. 468, 530-554.

Benotti, M.J., Trenholm, R.A., Vanderford, B.J., Holady, J.C., Stanford, B.D., Snyder, S.A., 2009. Pharmaceuticals and endocrine disrupting compounds in us drinking water. Environ. Sci. Technol. 43, 597-603.

Bollmann, U.E., Moler, A., Xie, Z.Y., Ebinghaus, R., Einax, J.W., 2012. Occurrence and fate of organophosphorus flame retardants and plasticizers in coastal and marine surface waters. Water Res. 46, 531-538.
Bradford, M.M., 1976. A rapid and sensitive method for the quantitation of microgram quantities of protein utilizing the principle of protein-dye binding. Anal. Biochem. 72, 248-254.

Campoy-López, Pedro, Pereira-Pinto, E., Mantilla-Aldana, L., Beiras, R., 2018. Estrogenic effects of an organophosphorous flame retardant (TCPP) on edible sea urchin "Paracentrotus lividus" L. Conference: SETAC Europe 28th Annual Meeting, Rome, Italy.

Cao, S., Zeng, X., Song, H., Li, H., Yu, Z., Sheng, G., et al., 2012. Levels and distributions of organophosphate flame retardants and plasticizers in sediment from taihu lake. China. Environ. Toxicol. Chem. 31, 1478-1484.

Casida, J.E., Quistad, G.B., 2005. Serine hydrolase targets of organophosphorus toxicants. Chem. Biol. Interact. 157, 277-283.

Cesar-Razquin, A., Snijder, B., Frappier-Brinton, T., Isserlin, R., Gyimesi, G., Bai, X., et al., 2015. A call for systematic research on solute carriers. Cell 162, 478-487.

Cesca, F., Baldelli, P., Valtorta, F., Benfenati, F., 2010. The synapsins: key actors of synapse function and plasticity. Prog. Neurobiol. 91, 313-348.

Chang, C.T., Chung, H.Y., Su, H.T., Tseng, H.P., Tzou, W.S., Hu, C.H., 2013. Regulation of zebrafish CYP3A65 transcription by AHR2. Toxicol. Appl. Pharmacol. 270, 174-184.

Chater, T.E., Goda, Y., 2014. The role of AMPA receptors in postsynaptic mechanisms of synaptic plasticity. Front. Cell. Neurosci. 8, 401.

Chedik, L., Bruyere, A., Fardel, O., 2019. Interactions of organophosphorus pesticides with solute carrier (slc) drug transporters. Xenobiotica 49, 363-374.

Costa, L.G., 1998. Signal transduction in environmental neurotoxicity. Annu. Rev. Pharmacol. 38, 21-43.

Crump, D., Chiu, S., Kennedy, S.W., 2012. Effects of tris(1,3-dichloro-2-propyl) phosphate and tris(1-chloropropyl) phosphate on cytotoxicity and mRNA expression in primary cultures of avian hepatocytes and neuronal cells. Toxicol. Sci. 126, $140-148$.

Danielson, P.B., 2002. The cytochrome P450 superfamily: biochemistry, evolution and drug metabolism in humans. Curr. Drug Metab. 3, 561-597.

Dishaw, L.V., Powers, C.M., Ryde, I.T., Roberts, S.C., Seidler, F.J., Slotkin, T.A., et al., 2011. Is the pentabde replacement, tris (1,3-dichloropropyl) phosphate (TDCPP), a developmental neurotoxicant? Studies in PC12 cells. Toxicol. Appl. Pharm. 256, 281-289.

Dishaw, L.V., Hunter, D.L., Padnos, B., Padilla, S., Stapleton, H.M., 2014. Developmental exposure to organophosphate flame retardants elicits overt toxicity and alters behavior in early life stage zebrafish (Danio rerio). Toxicol. Sci. 142, 445-454.

Dong, Y., Zhang, S., 2016. Ecological relevance of energy metabolism: transcriptional responses in energy sensing and expenditure to thermal and osmotic stresses in an intertidal limpet. Funct. Ecol. 30, 1539-1548.

Du, J., Li, H., Xu, S., Zhou, Q., Jin, M., Tang, J., 2019. A review of organophosphorus flame retardants (OPFRs): occurrence, bioaccumulation, toxicity, and organism exposure. Environ. Sci. Pollut. R. 26, 22126-22136.

Du, Z., Zhang, Y., Wang, G., Peng, J., Wang, Z., Gao, S., 2016. TPHP exposure disturbs carbohydrate metabolism, lipid metabolism, and the DNA damage repair system in zebrafish liver. Sci. Rep. 21827.

Environmental Protection Agency U.S., 2015. Flame Retardants Used in Flexible Polyurethane Foam: An Alternatives Assessment Update. www.epa.gov/sites/production/ files/2015-08/documents/ffr_final.pdf.

Farhat, A., Crump, D., Chiu, S., Williams, K.L., Letcher, R.J., Gauthier, L.T., Kennedy, S.W., 2013. In Ovo effects of two organophosphate flame retardants-TCPP and TDCPPon pipping success, development, mRNA expression, and thyroid hormone levels in chicken embryos. Toxicol. Sci. 134, 92-102.

Flaskos, J., 2014. The neuronal cytoskeleton as a potential target in the developmental neurotoxicity of organophosphorothionate insecticides. Basic Clin. Pharmacol. 115, 201-208.

Francavilla, C., Loeffler, S., Piccini, D., Kren, A., Christofori, G., Cavallaro, U., 2007. Neural cell adhesion molecule regulates the cellular response to fibroblast growth factor. J. Cell Sci. 120, 4388-4394.

Gu, Y., Yang, Y., Wan, B., Li, M., Guo, L., 2018. Inhibition of O-linked Nacetylglucosamine transferase activity in PC12 cells - a molecular mechanism of organophosphate flame retardants developmental neurotoxicity. Biochem. Pharmacol. 152, 21-33.

Harold, D., Abraham, R., Hollingworth, P., Sims, R., Gerrish, A., Hamshere, M.L., et al., 2009 Genome-wide association study identifies variants at CLU and PICALM associated with Alzheimer's disease. Nat. Genet. 41, 1088-1093.

Hartmann, P.C., Burgi, D., Giger, W., 2004. Organophosphate flame retardants and plasticizers in indoor air. Chemosphere 57, 781-787.

Hervé, J.C., Bourmeyster, N., 2015. Rho GTPases at the crossroad of signaling networks in mammals. Small GTPase 6, 43-48.

Hou, R., Xu, Y., Wang, Z., 2016. Review of OPFRs in animals and humans: absorption, bioaccumulation, metabolism, and internal exposure research. Chemosphere 153, 78-90.

Inazu, M., 2014. Choline transporter-like proteins ctls/slc44 family as a novel molecular target for cancer therapy. Biopharm. Drug Dispos. 35, 431-449.

Ji, C., Cao, L., Li, F., 2015. Toxicological evaluation of two pedigrees of clam Ruditapes philippinarum as bioindicators of heavy metal contaminants using metabolomics. Environ. Toxicol. Phar. 39, 545-554.

Ji, C., Lu, Z., Xu, L., Li, F., Cong, M., Shan, X., et al., 2019. Evaluation of mitochondrial toxicity of cadmium in clam Ruditapes philippinarum using iTRAQ-based proteomics. Environ. Pollut. 251, 802-810.

Lee, S., Cho, H.J., Choi, W., Moon, H.B., 2018. Organophosphate flame retardants (opfrs) in water and sediment: occurrence, distribution, and hotspots of contamination of lake shihwa, Korea. Mar. Pollut. Bull. 130, 105-112.

Lewis, J.A., Szilagyi, M., Gehman, E., Dennis, W.E., Jackson, D.A., 2009. Distinct patterns of gene and protein expression elicited by organophosphorus pesticides in Caenorhabditis elegans. BMC Genomics 10, 202. 
Li, J., Xie, Z., Mi, W., Lai, S., Tian, C., Emeis, K., et al., 2017. Organophosphate esters in air snow, and seawater in the North Atlantic and the Arctic. Environ. Sci. Technol. 51, 6887-6896.

Li, L., Chin, L.S., Shupliakov, O., Brodin, L., Sihra, T.S., Hvalby, O., Jensen, V., Zheng, D. McNamara, J.O., Greengard, P., 1995. Impairment of synaptic vesicle clustering and of synaptic transmission, and increased seizure propensity, in synapsin I-deficient mice. Natl. Acad. Sci. U. S. A. 92, 9235-9239.

i, L, Liu, X, You, L, Zhang L, Zhao, J, Wu, H. 2012. Uptake pathways and subcellula fractionation of cd in the polychaete Nereis diversicolor. Ecotoxicology 21, 104-110.

Meyer, J.N., Leung, M.C.K., Rooney, J.P., Sendoel, A., Hengartner, M.O., Kisby, G.E., Bess, A.S 2013. Mitochondria as a target of environmental toxicants. Toxicol. Sci. 134, 1-17.

Morimoto, K., Sato, K., Sato, S., Suemaru, S., Sato, T., Yamada, N., Hayabara, T., 1998. Increases in mRNA levels for synapsin I but not synapsin II in the hippocampus of the rat kindling model of epilepsy. Seizure 7, 229-235.

Nagao, K., Ono, K., Iwanaga, Y., Tamaki, Y., Kojima, Y., Horie, T., Nishi, H., Kinoshita, M. Kuwabara, Y., Hasegawa, K., Kita, T., Kimura, T., 2010. Neural cell adhesion molecule is a cardioprotective factor up-regulated by metabolic stress. J. Mol. Cell. Cardiol. 48 1157-1168.

Novak, J., Benisek, M., Hilscherova, K., 2008. Disruption of retinoid transport, metabolism and signaling by environmental pollutants. Environ. Int. 34, 898-913.

Olkkonen, V.M., Béaslas, O., Nissilä, E., 2012. Oxysterols and their cellular effectors. Biomolecules 2, 76-103.

Palosaari, P.M., Kilponen, J.M., Sormunen, R.T., Hassinen, I.E., Hiltunen, J.K., 1990. Delta-3, delta-2-enoyl-coa isomerases - characterization of the mitochondrial isoenzyme in the rat. J. Biol. Chem. 265, 3347-3353.

Persson, J., Wang, T., Hagberg, J., 2018. Organophosphate flame retardants and plasticizers in indoor dust, air and window wipes in newly built low-energy preschools. Sci. Total Environ. 628-629, 159-168.

Porcelli, V., Fiermonte, G., Longo, A., Palmieri, F., 2014. The human gene SLC25A29, of solute carrier family 25 , encodes a mitochondrial transporter of basic amino acids. J. Biol. Chem. 289, 13374-13384.

Poulson-Ellestad, K.L., Jones, C.M., Roy, J., Viant, M.R., Fernandez, F.M., Kubanek, J., et al., 2014. Metabolomics and proteomics reveal impacts of chemically mediated competition on marine plankton. P. Natl. Acad. Sci. U.S.A. 111, 9009-9014.

Reichardt, L.F., 2006. Neurotrophin-regulated signaling pathways. Philos. Trans. R. Soc Lond. Ser. B Biol. Sci. 361, 1545-1564.

Ren, Y., Zheng, J., Yao, X., Weng, G., Wu, L., 2014. Essential role of the cGMP/PKG signaling pathway in regulating the proliferation and survival of human renal carcinoma cells Int. J. Mol. Med. 34, 1430-1438.

Ross, M.K., Borazjani, A., Mangum, L.C., Wang, R., Crow, J.A., 2014. Effects of toxicologically relevant xenobiotics and the lipid-derived electrophile 4-hydroxynonenal on macrophage cholesterol efflux: silencing carboxylesterase 1 has paradoxical effects on cholesterol uptake and efflux. Chem. Res. Toxicol. 27, 1743-1756.

Sakai, K., Igarashi, M., Yamamuro, D., Ohshiro, T., Nagashima, S., Takahashi, M., et al., 2014 Critical role of neutral cholesteryl ester hydrolase 1 in cholesteryl ester hydrolysis in murine macrophages. J. Lipid Res. 55, 2033-2040.

Schroepfer, G.J., 2000. Oxysterols: modulators of cholesterol metabolism and other processes. Physiol. Rev. 80, 361-554.

Schulz, N., Himmelbauer, H., Rath, M., van Weeghel, M., Houten, S., Kulik, W., et al., 2011. Role of medium- and short-chain l-3-hydroxyacyl-coa dehydrogenase in the regulation of body weight and thermogenesis. Endocrinology 152, 4641-4651.
Sheng, L., Leshchyns'ka, I., Sytnyk, V., 2013. Cell adhesion and intracellular calcium signaling in neurons. Cell. Commun. Signal. 11, 94

Shoshan-Barmatza, V., De Pinto, V., Zweckstetter, M., Raviv, Z., Keinan, N., Arbela, N., 2010. VDAC, a multi-functional mitochondrial protein regulating cell life and death. Mol. Asp. Med. 31, 227-285.

Slotkin, T.A., Skavicus, S., Stapleton, H.M., Seidler, F.J., 2017. Brominated and organophosphate flame retardants target different neurodevelopmental stages, characterized with embryonic neural stem cells and neuronotypic PC12 cells. Toxicology 390, $32-42$.

Sokolova, I.M., 2013. Energy-limited tolerance to stress as a conceptual framework to integrate the effects of multiple stressors. Integr. Comp. Biol. 53, 597-608.

Sokolova, I.M., Sokolov, E.P., Ponnappa, K.M., 2005. Cadmium exposure affects mitochondrial bioenergetics and gene expression of key mitochondrial proteins in the eastern oyster Crassostrea virginica gmelin (bivalvia : Ostreidae). Aquat. Toxicol. 73, 242-255.

Sun, JX. Tang S, Peng. H., Saunders, D.MV Doering J. A, Hecker, M., et al, 2016a Combined transcriptomic and proteomic approach to identify toxicity pathways in early life stages of japanese medaka (Oryzias latipes) exposed to 1,2,5,6tetrabromocyclooctane (TBCO). Environ. Sci Technol. 50, 7781-7790.

Sun, L., Tan, H., Peng, T., Wang, S., Xu, W., Qian, H., et al., 2016b. Developmental neurotoxicity of organophosphate flame retardants in early life stages of japanese medaka (Oryzias latipes). Environ. Toxicol. Chem. 35, 2931-2940.

Sundkvist, A.M. Olofsson, U. Haglund, P. 2010. Organophosphorus flame retardants and plasticizers in marine and fresh water biota and in human milk. J. Environ. Monitor 12, 943-951

van der Veen, I., de Boer, J., 2012. Phosphorus flame retardants: properties, production, environmental occurrence, toxicity and analysis. Chemosphere 88, 1119-1153.

Wang, R., Tang, J., Xie, Z., Mi, W., Chen, Y., Wolschke, H., et al., 2015. Occurrence and spatial distribution of organophosphate ester flame retardants and plasticizers in 40 rivers draining into the bohai sea, north China. Environ. Pollut. 198, 172-178.

Xu, T., Li, P., Wu, S., Lei, L., He, D., 2017. Tris(2-chloroethyl) phosphate (TCEP) and tris(2chloropropyl) phosphate (TCPP) induce locomotor deficits and dopaminergic degeneration in Caenorhabditis elegans. Toxicol. Res. 6, 63-72.

Yamatani, H., Kawasaki, T., Mita, S., Inagaki, N., Hirata, T., 2010. Proteomics analysis of the temporal changes in axonal proteins during maturation. Dev. Neurobiol. 70, 523-537.

Yang, F., Ding, J., Huang, W., Xie, W., Liu, W., 2014. Particle size-specific distributions and preliminary exposure assessments of organophosphate flame retardants in office air particulate matter. Environ. Sci. Technol. 48, 63-70.

Yang, W., Zhao, F., Fang, Y., Li, L., Li, C., Ta, N., 2018. ${ }^{1} \mathrm{H}$-nuclear magnetic resonance metabolomics revealing the intrinsic relationships between neurochemical alterations and neurobehavioral and neuropathological abnormalities in rats exposed to tris(2chloroethyl)phosphate. Chemosphere 200, 649-659.

Zhang, B., Zhang, Y., Dagher, M., Shacter, E., 2005. Rho GDP dissociation inhibitor protects cancer cells against drug-induced apoptosis. Cancer Res. 65, 6054-6062.

Zhang, X., Xia, P., Wang, P., Yang, J., Baird, D., 2018. Omics advances in ecotoxicology. Environ. Sci. Technol. 52, 3842-3851.

Zhong, M., Tang, J., Mi, L., Li, F., Wang, R., Huang, G., et al., 2017. Occurrence and spatial distribution of organophosphorus flame retardants and plasticizers in the bohai and yellow seas, China. Mar. Pollut. Bull. 121, 331-338.

Zhou, Y., Danbolt, N.C., 2014. Glutamate as a neurotransmitter in the healthy brain. J. Neural Transm. 121, 799-817. 